Findings

Findings of the Association for Computational Linguistics

Findings of ACL: EMNLP 2021

November 7-11, 2021 
(C2021 The Association for Computational Linguistics

Order copies of this and other ACL proceedings from:

Association for Computational Linguistics (ACL)

209 N. Eighth Street

Stroudsburg, PA 18360

USA

Tel: +1-570-476-8006

Fax: +1-570-476-0860

acl@aclweb. org

ISBN 978-1-955917-10-0 


\section{Message from the Program Chairs}

Welcome to the Findings of ACL: EMNLP 2021! To continue the success of Findings of ACL series, we followed EMNLP 2020's initiative to produce this accompanying volume, for papers that narrowly missed acceptance to the main conference, but were judged to be solid, well-executed research, and worthy of publication. Out of the 3,600 submissions under review for EMNLP 2021, 445 papers were invited to be included in the Findings. 26 papers declined the offer, leading to 419 (300 long and 119 short) papers to be published in the Findings of ACL: EMNLP 2021. The papers accepted to Findings equates to $11.6 \%$ of the reviewed papers, giving an aggregate acceptance rate for EMNLP and Findings of $34.9 \%$. In addition, we also accepted 5 long papers to the Findings, through the first ACL Rolling Review (ARR) pilot at EMNLP 2021. These papers are published in this volume as any other Findings paper.

Papers published in Findings of ACL count as full publications. They are not assigned a presentation slot in the main conference, but rather are published online in a separate volume in the ACL Anthology. There are a number of motivations for this new publication, from allowing timely work to be published quickly, to being more accepting of solid work, and helping to manage the increasing reviewing burden on the community. To increase the visibility of the Findings papers, the authors are requested to submit a 6 (for long papers) or 3 minutes (for short papers) pre-recorded video presentation to be included in the virtual conference, hosted by Underline. Our workshop chairs also helped to pair Findings papers with EMNLP 2021 workshops, and as a result, around 100 Findings papers will be presented at those workshops.

The reviewing process for Findings is largely the same as for the main conference and accordingly we wish to thank all involved in EMNLP 2021 for their efforts, as detailed in the Preface to the Proceedings of EMNLP 2021. We would like to specifically thank:

- The whole Program Committee for reviewing the submissions, and in particular, the Senior Area Chairs for making recommendation on final paper decisions for Findings;

- The Ethics Committee, chaired by Margot Mieskes and Chris Potts, for their hard work to ensure that all the conditionally accepted Findings papers have addressed the ethical issues appropriately;

- Past *ACL PCs, including Trevor Cohn, Yulan He and Yang Liu (EMNLP 2020), and Fei Xia, Wenjie Li, Roberto Navigli (ACL-IJCNLP 2021) for sharing their experience on Findings papers;

- Findings Chairs Gabriel Stanovsky and Tim Van de Cruys, for their tremendous effort in making the volume of Findings of ACL: EMNLP 2021;

- Workshop Chairs Parisa Kordjamshidi and Minlie Huang, for connecting Findings paper authors with workshop organizers for possible presentations.

We hope that Findings will continue to serve as a companion to future conferences, and become an important venue for excellent, widely-read, and highly cited work in NLP.

Xuanjing Huang, Fudan University, China

Lucia Specia, Imperial College London, UK

Scott Wen-tau Yih, Facebook, USA

EMNLP 2021 Program Committee Co-Chairs 



\section{Table of Contents}

K-PLUG: Knowledge-injected Pre-trained Language Model for Natural Language Understanding and Generation in E-Commerce

Song Xu, Haoran Li, Peng Yuan, Yujia Wang, Youzheng Wu, Xiaodong He, Ying Liu and Bowen Zhou ...............................................................

Extracting Topics with Simultaneous Word Co-occurrence and Semantic Correlation Graphs: Neural Topic Modeling for Short Texts

Yiming Wang, Ximing Li, Xiaotang Zhou and Jihong Ouyang. . .18

Self-supervised Contrastive Cross-Modality Representation Learning for Spoken Question Answering

Chenyu You, Nuo Chen and Yuexian Zou ................................. 28

Language Clustering for Multilingual Named Entity Recognition

Kyle Shaffer

Neural News Recommendation with Collaborative News Encoding and Structural User Encoding

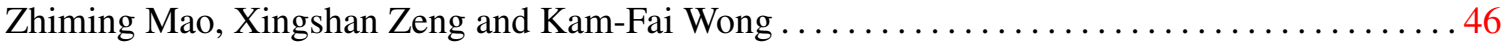

Self-Teaching Machines to Read and Comprehend with Large-Scale Multi-Subject Question-Answering Data

Dian Yu, Kai Sun, Dong Yu and Claire Cardie

A Web Scale Entity Extraction System

Xuanting Cai, Quanbin Ma, Jianyu Liu, Pan Li, Qi Zeng, Zhengkan Yang and Pushkar Tripathi .69

Joint Multimedia Event Extraction from Video and Article

Brian Chen, Xudong Lin, Christopher Thomas, Manling Li, Shoya Yoshida, Lovish Chum, Heng Ji

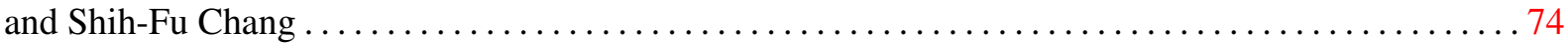

Fine-grained Semantic Alignment Network for Weakly Supervised Temporal Language Grounding

Yuechen Wang, Wengang Zhou and Houqiang Li .............................. 89

Factual Consistency Evaluation for Text Summarization via Counterfactual Estimation

Yuexiang Xie, Fei Sun, Yang Deng, Yaliang Li and Bolin Ding...

Cross-Modal Retrieval Augmentation for Multi-Modal Classification

Shir Gur, Natalia Neverova, Chris Stauffer, Ser-Nam Lim, Douwe Kiela and Austin Reiter ... . 111

HiTRANS: A Hierarchical Transformer Network for Nested Named Entity Recognition

Zhiwei Yang, Jing Ma, Hechang Chen, Yunke Zhang and Yi Chang .................. 124

Improving Embedding-based Large-scale Retrieval via Label Enhancement

Peiyang Liu, Xi Wang, Sen Wang, Wei Ye, Xiangyu Xi and Shikun Zhang .............. 133

Improving Privacy Guarantee and Efficiency of Latent Dirichlet Allocation Model Training Under Differential Privacy

Tao Huang and Hong Chen

Generating Mammography Reports from Multi-view Mammograms with BERT

Alexander Yalunin, Elena Sokolova, Ilya Burenko, Alexander Ponomarchuk, Olga Puchkova and

Dmitriy Umerenkov ................................................. 153 
Euphemistic Phrase Detection by Masked Language Model

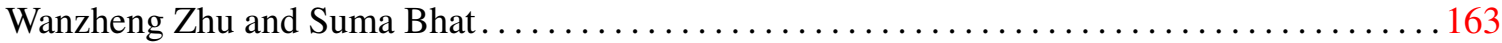

Decomposing Complex Questions Makes Multi-Hop QA Easier and More Interpretable

Ruiliu Fu, Han Wang, Xuejun Zhang, Jun Zhou and Yonghong Yan . . . . . . . . . . . . . . . . 169

Segmenting Natural Language Sentences via Lexical Unit Analysis

Yangming Li, Lemao Liu and Shuming Shi.

Dense Hierarchical Retrieval for Open-domain Question Answering

Ye Liu, Kazuma Hashimoto, Yingbo Zhou, Semih Yavuz, Caiming Xiong and Philip Yu ...... 188

Visually Grounded Concept Composition

Bowen Zhang, Hexiang Hu, Linlu Qiu, Peter Shaw and Fei Sha

Compositional Networks Enable Systematic Generalization for Grounded Language Understanding

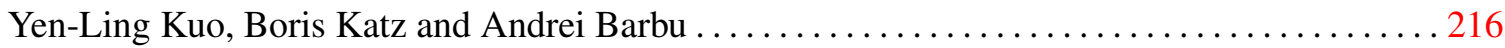

An Unsupervised Method for Building Sentence Simplification Corpora in Multiple Languages

Xinyu Lu, Jipeng Qiang, Yun Li, Yunhao Yuan and Yi Zhu ................... 227

WhiteningBERT: An Easy Unsupervised Sentence Embedding Approach

Junjie Huang, Duyu Tang, Wanjun Zhong, Shuai Lu, Linjun Shou, Ming Gong, Daxin Jiang and

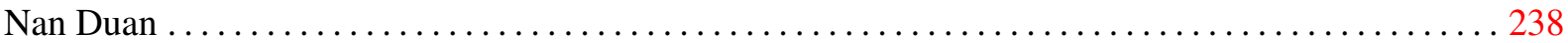

TWEETSUMM - A Dialog Summarization Dataset for Customer Service

Guy Feigenblat, Chulaka Gunasekara, Benjamin Sznajder, Sachindra Joshi, David Konopnicki and

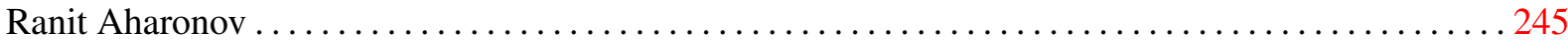

Discourse-Based Sentence Splitting

Liam Cripwell, Joël Legrand and Claire Gardent . . . . . . . . . . . . . . . . . . . . 261

Multi-Task Dense Retrieval via Model Uncertainty Fusion for Open-Domain Question Answering

Minghan Li, Ming Li, Kun Xiong and Jimmy Lin . . . . . . . . . . . . . . . . . 274

Mining the Cause of Political Decision-Making from Social Media: A Case Study of COVID-19 Policies across the US States

Zhijing Jin, Zeyu Peng, Tejas Vaidhya, Bernhard Schoelkopf and Rada Mihalcea..........288

Self-Attention Graph Residual Convolutional Networks for Event Detection with dependency relations

Anan Liu, Ning $\mathrm{Xu}$ and Haozhe Liu . . . . . . . . . . . . . . . . . . . . . . . . . . . . 302

Mixup Decoding for Diverse Machine Translation

Jicheng Li, Pengzhi Gao, Xuanfu Wu, Yang Feng, Zhongjun He, Hua Wu and Haifeng Wang . 312

An Alignment-Agnostic Model for Chinese Text Error Correction

Liying Zheng, Yue Deng, Weishun Song, Liang Xu and Jing Xiao

Reasoning Visual Dialog with Sparse Graph Learning and Knowledge Transfer

Gi-Cheon Kang, Junseok Park, Hwaran Lee, Byoung-Tak Zhang and Jin-Hwa Kim ...

Exploring Sentence Community for Document-Level Event Extraction

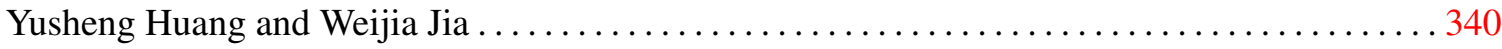


A Model of Cross-Lingual Knowledge-Grounded Response Generation for Open-Domain Dialogue Systems

San Kim, Jin Yea Jang, Minyoung Jung and Saim Shin 352

WHOSe Heritage: Classification of UNESCO World Heritage Statements of "Outstanding Universal Value" with Soft Labels

Nan Bai, Renqian Luo, Pirouz Nourian and Ana Pereira Roders

P-INT: A Path-based Interaction Model for Few-shot Knowledge Graph Completion

Jingwen Xu, Jing Zhang, Xirui Ke, Yuxiao Dong, Hong Chen, Cuiping Li and Yongbin Liu ... 385

Cartography Active Learning

Mike Zhang and Barbara Plank

Beyond Reptile: Meta-Learned Dot-Product Maximization between Gradients for Improved Single-Task Regularization

Akhil Kedia, Sai Chetan Chinthakindi and Wonho Ryu ...

GooAQ: Open Question Answering with Diverse Answer Types

Daniel Khashabi, Amos Ng, Tushar Khot, Ashish Sabharwal, Hannaneh Hajishirzi and Chris

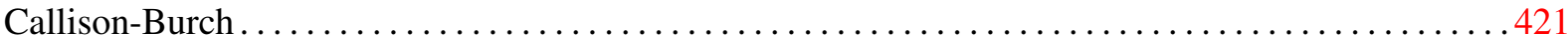

Attention Weights in Transformer NMT Fail Aligning Words Between Sequences but Largely Explain Model Predictions

Javier Ferrando and Marta R. Costa-jussà .................................. 434

BFClass: A Backdoor-free Text Classification Framework

Zichao Li, Dheeraj Mekala, Chengyu Dong and Jingbo Shang ..................... 444

Multilingual Chart-based Constituency Parse Extraction from Pre-trained Language Models

Taeuk Kim, Bowen Li and Sang-goo Lee.................................. 454

Hyperbolic Geometry is Not Necessary: Lightweight Euclidean-Based Models for Low-Dimensional Knowledge Graph Embeddings

Kai Wang, Yu Liu, Dan Lin and Michael Sheng...

CascadeBERT: Accelerating Inference of Pre-trained Language Models via Calibrated Complete Models Cascade

Lei Li, Yankai Lin, Deli Chen, Shuhuai Ren, Peng Li, Jie Zhou and Xu Sun

Semi-supervised Relation Extraction via Incremental Meta Self-Training

Xuming Hu, Chenwei Zhang, Fukun Ma, Chenyao Liu, Lijie Wen and Philip S. Yu. . 487

Keyphrase Generation with Fine-Grained Evaluation-Guided Reinforcement Learning

Yichao Luo, Yige Xu, Jiacheng Ye, Xipeng Qiu and Qi Zhang ...

Improving Knowledge Graph Embedding Using Affine Transformations of Entities Corresponding to Each Relation

Jinfa Yang, Yongjie Shi, Xin Tong, Robin Wang, Taiyan Chen and Xianghua Ying ... 508

Using Question Answering Rewards to Improve Abstractive Summarization

Chulaka Gunasekara, Guy Feigenblat, Benjamin Sznajder, Ranit Aharonov and Sachindra Joshi518

Effect Generation Based on Causal Reasoning

Feiteng Mu, Wenjie Li and Zhipeng Xie 
Distilling Word Meaning in Context from Pre-trained Language Models

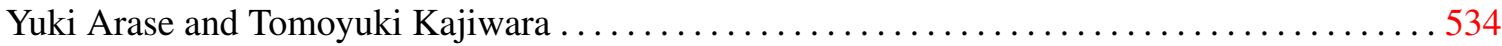

Unseen Entity Handling in Complex Question Answering over Knowledge Base via Language Generation

Xin Huang, Jung-Jae Kim and Bowei Zou .

Bidirectional Hierarchical Attention Networks based on Document-level Context for Emotion Cause Extraction

Guimin $\mathrm{Hu}$, Guangming Lu and Yi Zhao 558

Distantly Supervised Relation Extraction in Federated Settings

Dianbo Sui, Yubo Chen, Kang Liu and Jun Zhao

Casting the Same Sentiment Classification Problem

Erik Körner, Ahmad Dawar Hakimi, Gerhard Heyer and Martin Potthast . . .

Detecting Compositionally Out-of-Distribution Examples in Semantic Parsing

Denis Lukovnikov, Sina Daubener and Asja Fischer. .

Saliency-based Multi-View Mixed Language Training for Zero-shot Cross-lingual Classification

Siyu Lai, Hui Huang, Dong Jing, Yufeng Chen, Jinan Xu and Jian Liu ...

Fighting the COVID-19 Infodemic: Modeling the Perspective of Journalists, Fact-Checkers, Social Media Platforms, Policy Makers, and the Society

Firoj Alam, Shaden Shaar, Fahim Dalvi, Hassan Sajjad, Alex Nikolov, Hamdy Mubarak, Giovanni Da San Martino, Ahmed Abdelali, Nadir Durrani, Kareem Darwish, Abdulaziz Al-Homaid, Wajdi Zaghouani, Tommaso Caselli, Gijs Danoe, Friso Stolk, Britt Bruntink and Preslav Nakov .......... 611

FANATIC: FAst Noise-Aware TopIc Clustering

Ari Silburt, Anja Subasic, Evan Thompson, Carmeline Dsilva and Tarec Fares

Stream-level Latency Evaluation for Simultaneous Machine Translation

Javier Iranzo-Sánchez, Jorge Civera Saiz and Alfons Juan . . . . . . . . . . . . . . . . . 664

TSDAE: Using Transformer-based Sequential Denoising Auto-Encoderfor Unsupervised Sentence Embedding Learning

Kexin Wang, Nils Reimers and Iryna Gurevych

How Suitable Are Subword Segmentation Strategies for Translating Non-Concatenative Morphology?

Chantal Amrhein and Rico Sennrich.......................................... 689

Rethinking Why Intermediate-Task Fine-Tuning Works

Ting-Yun Chang and Chi-Jen Lu ....................................... 706

Learn Continually, Generalize Rapidly: Lifelong Knowledge Accumulation for Few-shot Learning

Xisen Jin, Bill Yuchen Lin, Mohammad Rostami and Xiang Ren .................... 714

Efficient Test Time Adapter Ensembling for Low-resource Language Varieties

Xinyi Wang, Yulia Tsvetkov, Sebastian Ruder and Graham Neubig .................... 730

An Analysis of Euclidean vs. Graph-Based Framing for Bilingual Lexicon Induction from Word Embedding Spaces

Kelly Marchisio, Youngser Park, Ali Saad-Eldin, Anton Alyakin, Kevin Duh, Carey Priebe and Philipp Koehn ................................................. 738 
How to Select One Among All ? An Empirical Study Towards the Robustness of Knowledge Distillation in Natural Language Understanding

Tianda Li, Ahmad Rashid, Aref Jafari, Pranav Sharma, Ali Ghodsi and Mehdi Rezagholizadeh 750

Recommend for a Reason: Unlocking the Power of Unsupervised Aspect-Sentiment Co-Extraction

Zeyu Li, Wei Cheng, Reema Kshetramade, John Houser, Haifeng Chen and Wei Wang ...... 763

Learning Hard Retrieval Decoder Attention for Transformers

Hongfei Xu, Qiuhui Liu, Josef van Genabith and Deyi Xiong

Recall and Learn: A Memory-augmented Solver for Math Word Problems

Shifeng Huang, Jiawei Wang, Jiao Xu, Da Cao and Ming Yang ...................... 786

An Uncertainty-Aware Encoder for Aspect Detection

Thi-Nhung Nguyen, Kiem-Hieu Nguyen, Young-In Song and Tuan-Dung Cao ............. 797

Improving Empathetic Response Generation by Recognizing Emotion Cause in Conversations

Jun Gao, Yuhan Liu, Haolin Deng, Wei Wang, Yu Cao, Jiachen Du and Ruifeng Xu . . . . . . . 807

Probing Across Time: What Does RoBERTa Know and When?

Zeyu Liu, Yizhong Wang, Jungo Kasai, Hannaneh Hajishirzi and Noah A. Smith............ 820

Knowledge-Guided Paraphrase Identification

Haoyu Wang, Fenglong Ma, Yaqing Wang and Jing Gao ...

R2-D2: A Modular Baseline for Open-Domain Question Answering

Martin Fajcik, Martin Docekal, Karel Ondrej and Pavel Smrz ....................... 854

What Does Your Smile Mean? Jointly Detecting Multi-Modal Sarcasm and Sentiment Using Quantum Probability

Yaochen Liu, Yazhou Zhang, Qiuchi Li, Benyou Wang and Dawei Song . .

Discovering Representation Sprachbund For Multilingual Pre-Training

Yimin Fan, Yaobo Liang, Alexandre Muzio, Hany Hassan, Houqiang Li, Ming Zhou and Nan Duan 881

Plan-then-Generate: Controlled Data-to-Text Generation via Planning

Yixuan Su, David Vandyke, Sihui Wang, Yimai Fang and Nigel Collier ...

Few-Shot Table-to-Text Generation with Prototype Memory

Yixuan Su, Zaiqiao Meng, Simon Baker and Nigel Collier

Leveraging Word-Formation Knowledge for Chinese Word Sense Disambiguation

Hua Zheng, Lei Li, Damai Dai, Deli Chen, Tianyu Liu, Xu Sun and Yang Liu ............. 918

Exploiting Curriculum Learning in Unsupervised Neural Machine Translation

Jinliang Lu and Jiajun Zhang ...

Robust Fragment-Based Framework for Cross-lingual Sentence Retrieval

Nattapol Trijakwanich, Peerat Limkonchotiwat, Raheem Sarwar, Wannaphong Phatthiyaphaibun,

Ekapol Chuangsuwanich and Sarana Nutanong ................................... 935

Towards Improving Adversarial Training of NLP Models

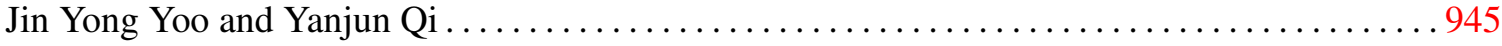


To Protect and To Serve? Analyzing Entity-Centric Framing of Police Violence

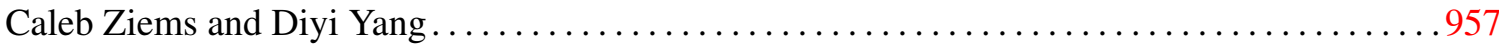

Calibrate your listeners! Robust communication-based training for pragmatic speakers

Rose Wang, Julia White, Jesse Mu and Noah Goodman. . . . . . . . . . . . . . . . . . . . . 977

When Retriever-Reader Meets Scenario-Based Multiple-Choice Questions

ZiXian Huang, Ao Wu, Yulin Shen, Gong Cheng and Yuzhong Qu ................... 985

Structured abbreviation expansion in context

Kyle Gorman, Christo Kirov, Brian Roark and Richard Sproat........................ 995

Task-adaptive Pre-training and Self-training are Complementary for Natural Language Understanding

Shiyang Li, Semih Yavuz, Wenhu Chen and Xifeng Yan.......................... 1006

CNNBiF: CNN-based Bigram Features for Named Entity Recognition

Chul Sung, Vaibhava Goel, Etienne Marcheret, Steven Rennie and David Nahamoo ......... 1016

Compositional Generalization via Semantic Tagging

Hao Zheng and Mirella Lapata..

Towards Document-Level Paraphrase Generation with Sentence Rewriting and Reordering

Zhe Lin, Yitao Cai and Xiaojun Wan

Exploring Decomposition for Table-based Fact Verification

Xiaoyu Yang and Xiaodan Zhu

Diversity and Consistency: Exploring Visual Question-Answer Pair Generation

Sen Yang, Qingyu Zhou, Dawei Feng, Yang Liu, Chao Li, Yunbo Cao and Dongsheng Li . . . 1053

Entity-level Cross-modal Learning Improves Multi-modal Machine Translation

Xin Huang, Jiajun Zhang and Chengqing Zong ...

Learning to Ground Visual Objects for Visual Dialog

Feilong Chen, Xiuyi Chen, Can Xu and Daxin Jiang

KERS: A Knowledge-Enhanced Framework for Recommendation Dialog Systems with Multiple Subgoals

Jun Zhang, Yan Yang, Chencai Chen, Liang He and Zhou Yu ....................... 1092

Less Is More: Domain Adaptation with Lottery Ticket for Reading Comprehension

Haichao Zhu, Zekun Wang, Heng Zhang, Ming Liu, Sendong Zhao and Bing Qin ...

Effectiveness of Pre-training for Few-shot Intent Classification

Haode Zhang, Yuwei Zhang, Li-Ming Zhan, Jiaxin Chen, Guangyuan Shi, Xiao-Ming Wu and Albert Y.S. Lam . . . . . . . . . . . . . . . . . . . . . . . . . . . . . . . . . . . . . . . . . . . . . . . . . 1114

Improving Abstractive Dialogue Summarization with Hierarchical Pretraining and Topic Segment

MengNan Qi, Hao Liu, YuZhuo Fu and Ting Liu ............................ 1121

Learning to Answer Psychological Questionnaire for Personality Detection

Feifan Yang, Tao Yang, Xiaojun Quan and Qinliang Su...

Exploiting Reasoning Chains for Multi-hop Science Question Answering

Weiwen Xu, Yang Deng, Huihui Zhang, Deng Cai and Wai Lam 
Winnowing Knowledge for Multi-choice Question Answering

Yeqiu Li, Bowei Zou, Zhifeng Li, Ai Ti Aw, Yu Hong and Qiaoming Zhu

Neural Media Bias Detection Using Distant Supervision With BABE - Bias Annotations By Experts

Timo Spinde, Manuel Plank, Jan-David Krieger, Terry Ruas, Bela Gipp and Akiko Aizawa . . 1166

Learning and Evaluating a Differentially Private Pre-trained Language Model

Shlomo Hoory, Amir Feder, Avichai Tendler, Sofia Erell, Alon Peled-Cohen, Itay Laish, Hootan Nakhost, Uri Stemmer, Ayelet Benjamini, Avinatan Hassidim and Yossi Matias .. 1178

Simulated Chats for Building Dialog Systems: Learning to Generate Conversations from Instructions

Biswesh Mohapatra, Gaurav Pandey, Danish Contractor and Sachindra Joshi ............. 1190

Past, Present, and Future: Conversational Emotion Recognition through Structural Modeling of Psychological Knowledge

Jiangnan Li, Zheng Lin, Peng Fu and Weiping Wang . .

An unsupervised framework for tracing textual sources of moral change

Aida Ramezani, Zining Zhu, Frank Rudzicz and Yang Xu

Topic-Aware Contrastive Learning for Abstractive Dialogue Summarization

Junpeng Liu, Yanyan Zou, Hainan Zhang, Hongshen Chen, Zhuoye Ding, Caixia Yuan and Xiaojie

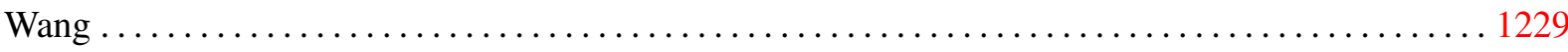

TWT: Table with Written Text for Controlled Data-to-Text Generation

Tongliang Li, Lei Fang, Jian-Guang Lou and Zhoujun Li

ArabicTransformer: Efficient Large Arabic Language Model with Funnel Transformer and ELECTRA Objective

Sultan Alrowili and Vijay Shanker 1255

Which is Making the Contribution: Modulating Unimodal and Cross-modal Dynamics for Multimodal Sentiment Analysis

Ying Zeng, Sijie Mai and Haifeng Hu

CVAE-based Re-anchoring for Implicit Discourse Relation Classification

Zujun Dou, Yu Hong, Yu Sun and Guodong Zhou.

Combining Curriculum Learning and Knowledge Distillation for Dialogue Generation

Qingqing Zhu, Xiuying Chen, Pengfei Wu, JunFei Liu and Dongyan Zhao .............. 1284

Improving End-to-End Task-Oriented Dialog System with A Simple Auxiliary Task

Yohan Lee

EDTC: A Corpus for Discourse-Level Topic Chain Parsing

Longyin Zhang, Xin Tan, Fang Kong and Guodong Zhou .

Multilingual Neural Machine Translation: Can Linguistic Hierarchies Help?

Fahimeh Saleh, Wray Buntine, Gholamreza Haffari and Lan Du.

Self Question-answering: Aspect-based Sentiment Analysis by Role Flipped Machine Reading Comprehension

Guoxin Yu, Jiwei Li, Ling Luo, Yuxian Meng, Xiang Ao and Qing He

Generalization in Text-based Games via Hierarchical Reinforcement Learning

Yunqiu Xu, Meng Fang, Ling Chen, Yali Du and Chengqi Zhang . . 
A Finer-grain Universal Dialogue Semantic Structures based Model For Abstractive Dialogue Summarization

Yuejie Lei, Fujia Zheng, Yuanmeng Yan, Keqing He and Weiran Xu .................. 1354

Constructing contrastive samples via summarization for text classification with limited annotations

Yangkai Du, Tengfei Ma, Lingfei Wu, Fangli Xu, Xuhong Zhang, Bo Long and Shouling Ji . 1365

End-to-end Neural Information Status Classification

Yufang Hou .

EventKE: Event-Enhanced Knowledge Graph Embedding

Zixuan Zhang, Hongwei Wang, Han Zhao, Hanghang Tong and Heng Ji ...

Modeling Concentrated Cross-Attention for Neural Machine Translation with Gaussian Mixture Model

Shaolei Zhang and Yang Feng ....................................... 1401

Inconsistency Matters: A Knowledge-guided Dual-inconsistency Network for Multi-modal Rumor Detection

Mengzhu Sun, Xi Zhang, Jianqiang Ma and Yazheng Liu . 1412

EfficientBERT: Progressively Searching Multilayer Perceptron via Warm-up Knowledge Distillation

Chenhe Dong, Guangrun Wang, Hang Xu, Jiefeng Peng, Xiaozhe Ren and Xiaodan Liang ... 1424

Uni-FedRec: A Unified Privacy-Preserving News Recommendation Framework for Model Training and Online Serving

Tao Qi, Fangzhao Wu, Chuhan Wu, Yongfeng Huang and Xing Xie. ...

Mapping Language to Programs using Multiple Reward Components with Inverse Reinforcement Learning

Sayan Ghosh and Shashank Srivastava...

Topic-Guided Abstractive Multi-Document Summarization

Peng Cui and $\mathrm{Le} \mathrm{Hu}$

An Edge-Enhanced Hierarchical Graph-to-Tree Network for Math Word Problem Solving

Qinzhuo Wu, Qi Zhang and Zhongyu Wei ...

SciXGen: A Scientific Paper Dataset for Context-Aware Text Generation

Hong Chen, Hiroya Takamura and Hideki Nakayama ...

Don't Miss the Potential Customers! Retrieving Similar Ads to Improve User Targeting

Yi Feng, Ting Wang, Chuanyi Li, Vincent Ng, Jidong Ge, Bin Luo, Yucheng Hu and Xiaopeng Zhang.

Cross-lingual Transfer for Text Classification with Dictionary-based Heterogeneous Graph

Nuttapong Chairatanakul, Noppayut Sriwatanasakdi, Nontawat Charoenphakdee, Xin Liu and Tsuyoshi Murata . . . 1504

Improving Distantly-Supervised Named Entity Recognition with Self-Collaborative Denoising Learning Xinghua Zhang, Bowen Yu, Tingwen Liu, Zhenyu Zhang, Jiawei Sheng, Xue Mengge and Hongbo

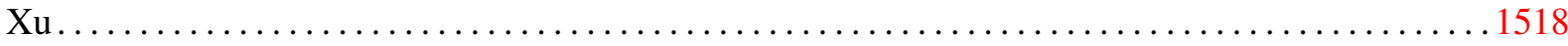

Entity-Based Semantic Adequacy for Data-to-Text Generation

Juliette Faille, Albert Gatt and Claire Gardent 
MiRANews: Dataset and Benchmarks for Multi-Resource-Assisted News Summarization

Xinnuo Xu, Ondřej Dušek, Shashi Narayan, Verena Rieser and Ioannis Konstas............ 1541

A Conditional Generative Matching Model for Multi-lingual Reply Suggestion

Budhaditya Deb, Guoqing Zheng, Milad Shokouhi and Ahmed Hassan Awadallah ...

Rethinking Sentiment Style Transfer

Ping Yu, Yang Zhao, Chunyuan Li and Changyou Chen

HypoGen: Hyperbole Generation with Commonsense and Counterfactual Knowledge

Yufei Tian, Arvind krishna Sridhar and Nanyun Peng

Profiling News Discourse Structure Using Explicit Subtopic Structures Guided Critics

Prafulla Kumar Choubey and Ruihong Huang ...

ProtoInfoMax: Prototypical Networks with Mutual Information Maximization for Out-of-Domain Detection

Iftitahu Nimah, Meng Fang, Vlado Menkovski and Mykola Pechenizkiy 1606

Learning from Language Description: Low-shot Named Entity Recognition via Decomposed Framework

Yaqing Wang, Haoda Chu, Chao Zhang and Jing Gao 1618

BERT might be Overkill: A Tiny but Effective Biomedical Entity Linker based on Residual Convolutional Neural Networks

Tuan Lai, Heng Ji and ChengXiang Zhai ....

Char2Subword: Extending the Subword Embedding Space Using Robust Character Compositionality

Gustavo Aguilar, Bryan McCann, Tong Niu, Nazneen Rajani, Nitish Shirish Keskar and Thamar

Solorio 1640

Exploring Multitask Learning for Low-Resource Abstractive Summarization

Ahmed Magooda, Diane Litman and Mohamed Elaraby ...

Conical Classification For Efficient One-Class Topic Determination

Sameer Khanna 1662

Improving Dialogue State Tracking with Turn-based Loss Function and Sequential Data Augmentation

Jarana Manotumruksa, Jeff Dalton, Edgar Meij and Emine Yilmaz ................... 1674

TIAGE: A Benchmark for Topic-Shift Aware Dialog Modeling

Huiyuan Xie, Zhenghao Liu, Chenyan Xiong, Zhiyuan Liu and Ann Copestake ............ 1684

Optimal Neural Program Synthesis from Multimodal Specifications

Xi Ye, Qiaochu Chen, Isil Dillig and Greg Durrett

Sent2Span: Span Detection for PICO Extraction in the Biomedical Text without Span Annotations

Shifeng Liu, Yifang Sun, Bing Li, Wei Wang, Florence T. Bourgeois and Adam G. Dunn .... 1705

When in Doubt: Improving Classification Performance with Alternating Normalization

Menglin Jia, Austin Reiter, Ser-Nam Lim, Yoav Artzi and Claire Cardie ............... 1716

APGN: Adversarial and Parameter Generation Networks for Multi-Source Cross-Domain Dependency Parsing

Ying Li, Meishan Zhang, Zhenghua Li, Min Zhang, Zhefeng Wang, baoxing Huai and Nicholas

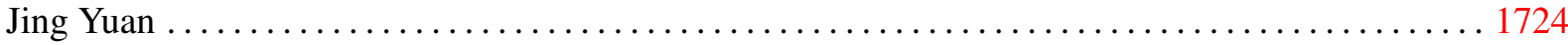


"Let Your Characters Tell Their Story": A Dataset for Character-Centric Narrative Understanding

Faeze Brahman, Meng Huang, Oyvind Tafjord, Chao Zhao, Mrinmaya Sachan and Snigdha Chaturvedi 1734

Towards Developing a Multilingual and Code-Mixed Visual Question Answering System by Knowledge Distillation

Humair Raj Khan, Deepak Gupta and Asif Ekbal

An Iterative Multi-Knowledge Transfer Network for Aspect-Based Sentiment Analysis

Yunlong Liang, Fandong Meng, Jinchao Zhang, Yufeng Chen, Jinan Xu and Jie Zhou ...

Semantic Alignment with Calibrated Similarity for Multilingual Sentence Embedding

Jiyeon Ham and Eun-Sol Kim ......................................... 1781

fBERT: A Neural Transformer for Identifying Offensive Content

Diptanu Sarkar, Marcos Zampieri, Tharindu Ranasinghe and Alexander Ororbia ...

WIKIBIAS: Detecting Multi-Span Subjective Biases in Language

Yang Zhong, Jingfeng Yang, Wei Xu and Diyi Yang. .

UnClE: Explicitly Leveraging Semantic Similarity to Reduce the Parameters of Word Embeddings

Zhi Li, Yuchen Zhai, Chengyu Wang, Minghui Qiu, Kailiang Li and Yin Zhang........... 1815

Grounded Graph Decoding improves Compositional Generalization in Question Answering

Yu Gai, Paras Jain, Wendi Zhang, Joseph Gonzalez, Dawn Song and Ion Stoica. . .

Enhancing Visual Dialog Questioner with Entity-based Strategy Learning and Augmented Guesser

Duo Zheng, Zipeng Xu, Fandong Meng, Xiaojie Wang, Jiaan Wang and Jie Zhou .......... 1839

A Pretraining Numerical Reasoning Model for Ordinal Constrained Question Answering on Knowledge Base

Yu Feng, Jing Zhang, Gaole He, Wayne Xin Zhao, Lemao Liu, Quan Liu, Cuiping Li and Hong Chen

RoR: Read-over-Read for Long Document Machine Reading Comprehension

Jing Zhao, Junwei Bao, Yifan Wang, Yongwei Zhou, Youzheng Wu, Xiaodong He and Bowen Zhou 1862

Span Pointer Networks for Non-Autoregressive Task-Oriented Semantic Parsing

Akshat Shrivastava, Pierce Chuang, Arun Babu, Shrey Desai, Abhinav Arora, Alexander Zotov and

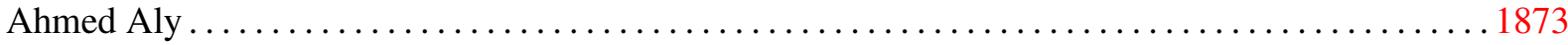

Language Resource Efficient Learning for Captioning

Jia Chen, Yike Wu, Shiwan Zhao and Qin Jin ...

Translation as Cross-Domain Knowledge: Attention Augmentation for Unsupervised Cross-Domain Segmenting and Labeling Tasks

Ruixuan Luo, Yi Zhang, Sishuo Chen and Xu Sun ...

ContractNLI: A Dataset for Document-level Natural Language Inference for Contracts

Yuta Koreeda and Christopher Manning .................................. 1907

Japanese Zero Anaphora Resolution Can Benefit from Parallel Texts Through Neural Transfer Learning

Masato Umakoshi, Yugo Murawaki and Sadao Kurohashi 
Grouped-Attention for Content-Selection and Content-Plan Generation

Bayu Distiawan Trisedya, Xiaojie Wang, Jianzhong Qi, Rui Zhang and Qingjun Cui ...

An Explicit-Joint and Supervised-Contrastive Learning Framework for Few-Shot Intent Classification and Slot Filling

Han Liu, Feng Zhang, Xiaotong Zhang, Siyang Zhao and Xianchao Zhang

Retrieve, Discriminate and Rewrite: A Simple and Effective Framework for Obtaining Affective Response in Retrieval-Based Chatbots

Xin Lu, Yijian Tian, Yanyan Zhao and Bing Qin ....

Span Fine-tuning for Pre-trained Language Models

Rongzhou Bao, Zhuosheng Zhang and Hai Zhao

DIRECT: Direct and Indirect Responses in Conversational Text Corpus

Junya Takayama, Tomoyuki Kajiwara and Yuki Arase

Retrieval, Analogy, and Composition: A framework for Compositional Generalization in Image Captioning

Zhan Shi, Hui Liu, Martin Renqiang Min, Christopher Malon, Li Erran Li and Xiaodan Zhu . 1990

TURINGBENCH: A Benchmark Environment for Turing Test in the Age of Neural Text Generation

Adaku Uchendu, Zeyu Ma, Thai Le, Rui Zhang and Dongwon Lee .................. 2001

Say 'YES' to Positivity: Detecting Toxic Language in Workplace Communications

Meghana Moorthy Bhat, saghar Hosseini, Ahmed Hassan Awadallah, Paul Bennett and Weisheng $\mathrm{Li}$ .. 2017

Natural SQL: Making SQL Easier to Infer from Natural Language Specifications

Yujian Gan, Xinyun Chen, Jinxia Xie, Matthew Purver, John R. Woodward, John Drake and Qiaofu

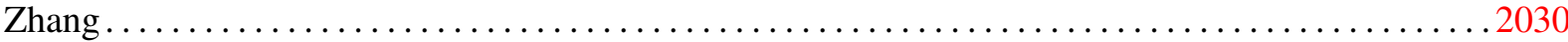

Mitigating Data Scarceness through Data Synthesis, Augmentation and Curriculum for Abstractive Summarization

Ahmed Magooda and Diane Litman .

Self- and Pseudo-self-supervised Prediction of Speaker and Key-utterance for Multi-party Dialogue Reading Comprehension

Yiyang Li and Hai Zhao. .

Few-Shot Novel Concept Learning for Semantic Parsing

Soham Dan, Osbert Bastani and Dan Roth ................................ 2064

Compositional Data and Task Augmentation for Instruction Following

Soham Dan, Xinran Han and Dan Roth

Are Factuality Checkers Reliable? Adversarial Meta-evaluation of Factuality in Summarization

Yiran Chen, Pengfei Liu and Xipeng Qiu ................................. 2082

On the Effects of Transformer Size on In- and Out-of-Domain Calibration

Soham Dan and Dan Roth.............................................. 2096

Detecting Polarized Topics Using Partisanship-aware Contextualized Topic Embeddings

Zihao He, Negar Mokhberian, António Câmara, Andres Abeliuk and Kristina Lerman....... 2102 
GenerativeRE: Incorporating a Novel Copy Mechanism and Pretrained Model for Joint Entity and Relation Extraction

Jiarun Cao and Sophia Ananiadou...

Re-entry Prediction for Online Conversations via Self-Supervised Learning

Lingzhi Wang, Xingshan Zeng, Huang Hu, Kam-Fai Wong and Daxin Jiang. .

proScript: Partially Ordered Scripts Generation

Keisuke Sakaguchi, Chandra Bhagavatula, Ronan Le Bras, Niket Tandon, Peter Clark and Yejin Choi

Speaker Turn Modeling for Dialogue Act Classification

Zihao He, Leili Tavabi, Kristina Lerman and Mohammad Soleymani

Unsupervised Domain Adaptation Method with Semantic-Structural Alignment for Dependency Parsing

Boda Lin, Mingzheng Li, Si Li and Yong Luo

Devil's Advocate: Novel Boosting Ensemble Method from Psychological Findings for Text Classification

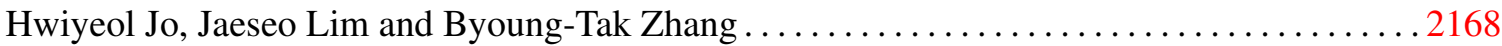

SideControl: Controlled Open-domain Dialogue Generation via Additive Side Networks

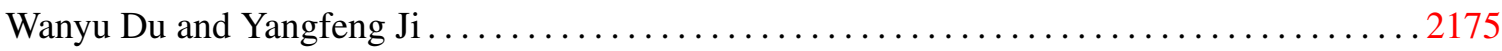

Is BERT a Cross-Disciplinary Knowledge Learner? A Surprising Finding of Pre-trained Models' Transferability

Wei-Tsung Kao and Hung-yi Lee

Geo-BERT Pre-training Model for Query Rewriting in POI Search

Xiao Liu, Juan Hu, Qi Shen and Huan Chen ...

Leveraging Bidding Graphs for Advertiser-Aware Relevance Modeling in Sponsored Search

Shuxian Bi, Chaozhuo Li, Xiao Han, Zheng Liu, Xing Xie, Haizhen Huang and Zengxuan Wen 2215

GPT3Mix: Leveraging Large-scale Language Models for Text Augmentation

Kang Min Yoo, Dongju Park, Jaewook Kang, Sang-Woo Lee and Woomyoung Park........ 2225

Context-aware Entity Typing in Knowledge Graphs

Weiran Pan, Wei Wei and Xian-Ling Mao

Attribute Alignment: Controlling Text Generation from Pre-trained Language Models

Dian Yu, Zhou Yu and Kenji Sagae. .

Generate \& Rank: A Multi-task Framework for Math Word Problems

Jianhao Shen, Yichun Yin, Lin Li, Lifeng Shang, Xin Jiang, Ming Zhang and Qun Liu ...... 2269

MIRTT: Learning Multimodal Interaction Representations from Trilinear Transformers for Visual Question Answering

Junjie Wang, Yatai Ji, Jiaqi Sun, Yujiu Yang and Tetsuya Sakai . .

UniteD-SRL: A Unified Dataset for Span- and Dependency-Based Multilingual and Cross-Lingual Semantic Role Labeling

Rocco Tripodi, Simone Conia and Roberto Navigli 
Enhancing Dual-Encoders with Question and Answer Cross-Embeddings for Answer Retrieval

Yanmeng Wang, Jun Bai, Ye Wang, Jianfei Zhang, Wenge Rong, Zongcheng Ji, Shaojun Wang and

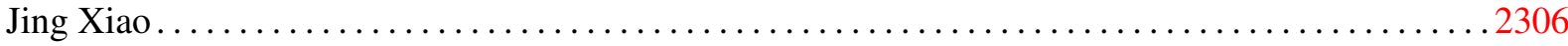

A Neural Graph-based Local Coherence Model

Mohsen Mesgar, Leonardo F. R. Ribeiro and Iryna Gurevych ...................... 2316

GiBERT: Enhancing BERT with Linguistic Information using a Lightweight Gated Injection Method

Nicole Peinelt, Marek Rei and Maria Liakata .............................. 2322

RollingLDA: An Update Algorithm of Latent Dirichlet Allocation to Construct Consistent Time Series from Textual Data

Jonas Rieger, Carsten Jentsch and Jörg Rahnenführer .......................... 2337

What If Sentence-hood is Hard to Define: A Case Study in Chinese Reading Comprehension

Jiawei Wang, Hai Zhao, Yinggong Zhao and Libin Shen ........................ 2348

Refining BERT Embeddings for Document Hashing via Mutual Information Maximization

Zijing Ou, Qinliang Su, Jianxing Yu, Ruihui Zhao, Yefeng Zheng and Bang Liu .......... 2360

REBEL: Relation Extraction By End-to-end Language generation

Pere-Lluís Huguet Cabot and Roberto Navigli ................................ 2370

Wine is not $v i n$. On the Compatibility of Tokenizations across Languages

Antonis Maronikolakis, Philipp Dufter and Hinrich Schütze ........................ 2382

Temporal Adaptation of BERT and Performance on Downstream Document Classification: Insights from Social Media

Paul Röttger and Janet Pierrehumbert. . .

Skim-Attention: Learning to Focus via Document Layout

Laura Nguyen, Thomas Scialom, Jacopo Staiano and Benjamin Piwowarski

Attention-based Contrastive Learning for Winograd Schemas

Tassilo Klein and Moin Nabi

Give the Truth: Incorporate Semantic Slot into Abstractive Dialogue Summarization

Lulu Zhao, Weihao Zeng, Weiran Xu and Jun Guo ...

Challenges in Detoxifying Language Models

Johannes Welbl, Amelia Glaese, Jonathan Uesato, Sumanth Dathathri, John Mellor, Lisa Anne Hendricks, Kirsty Anderson, Pushmeet Kohli, Ben Coppin and Po-Sen Huang. . . . . . . . . . . . . . 2447

Collecting a Large-Scale Gender Bias Dataset for Coreference Resolution and Machine Translation

Shahar Levy, Koren Lazar and Gabriel Stanovsky ............................. 2470

Competence-based Curriculum Learning for Multilingual Machine Translation

Mingliang Zhang, Fandong Meng, Yunhai Tong and Jie Zhou . . .

Informed Sampling for Diversity in Concept-to-Text NLG

Giulio Zhou and Gerasimos Lampouras .

Novel Natural Language Summarization of Program Code via Leveraging Multiple Input Representations

Fuxiang Chen, Mijung Kim and Jaegul Choo... 2510 
WikiNEuRal: Combined Neural and Knowledge-based Silver Data Creation for Multilingual NER

Simone Tedeschi, Valentino Maiorca, Niccolò Campolungo, Francesco Cecconi and Roberto Nav-

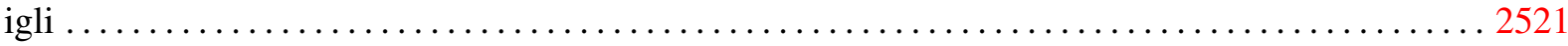

Beyond Grammatical Error Correction: Improving L1-influenced research writing in English using pretrained encoder-decoder models

Gustavo Zomer and Ana Frankenberg-Garcia...

Classification and Geotemporal Analysis of Quality-of-Life Issues in Tenant Reviews

Adam Haber and Zeev Waks.

Probing Pre-trained Language Models for Semantic Attributes and their Values

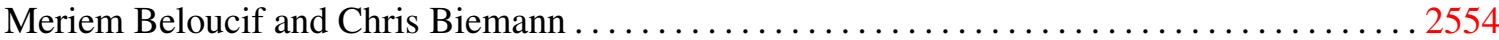

Uncovering the Limits of Text-based Emotion Detection

Nurudin Alvarez-Gonzalez, Andreas Kaltenbrunner and Vicenç Gómez................ 2560

Named Entity Recognition for Entity Linking: What Works and What's Next

Simone Tedeschi, Simone Conia, Francesco Cecconi and Roberto Navigli . . . .2584

Learning Numeracy: A Simple Yet Effective Number Embedding Approach Using Knowledge Graph

Hanyu Duan, Yi Yang and Kar Yan Tam

Weakly Supervised Semantic Parsing by Learning from Mistakes

Jiaqi Guo, Jian-Guang Lou, Ting Liu and Dongmei Zhang. . .

CodeQA: A Question Answering Dataset for Source Code Comprehension

Chenxiao Liu and Xiaojun Wan

Subword Mapping and Anchoring across Languages

Giorgos Vernikos and Andrei Popescu-Belis

CDLM: Cross-Document Language Modeling

Avi Caciularu, Arman Cohan, Iz Beltagy, Matthew Peters, Arie Cattan and Ido Dagan 2648

Patterns of Polysemy and Homonymy in Contextualised Language Models

Janosch Haber and Massimo Poesio

Cross-Lingual Leveled Reading Based on Language-Invariant Features

Simin Rao, Hua Zheng and Sujian Li ...

Controlled Neural Sentence-Level Reframing of News Articles

Wei-Fan Chen, Khalid Al Khatib, Benno Stein and Henning Wachsmuth

DialogueTRM: Exploring Multi-Modal Emotional Dynamics in a Conversation

Yuzhao Mao, Guang Liu, Xiaojie Wang, Weiguo Gao and Xuan Li ...

Adversarial Examples for Evaluating Math Word Problem Solvers

Vivek Kumar, Rishabh Maheshwary and Vikram Pudi

Improving Numerical Reasoning Skills in the Modular Approach for Complex Question Answering on Text

Xiao-Yu Guo, Yuan-Fang Li and Gholamreza Haffari

Retrieval Augmented Code Generation and Summarization

Md Rizwan Parvez, Wasi Ahmad, Saikat Chakraborty, Baishakhi Ray and Kai-Wei Chang. .. 2719 
Multilingual Translation via Grafting Pre-trained Language Models

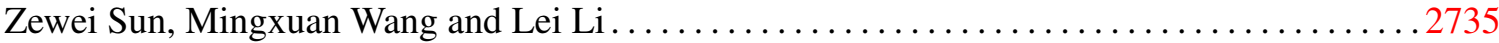

AEDA: An Easier Data Augmentation Technique for Text Classification

Akbar Karimi, Leonardo Rossi and Andrea Prati

A Comprehensive Comparison of Word Embeddings in Event \& Entity Coreference Resolution.

Judicael Poumay and Ashwin Ittoo . . . . . . . . . . . . . . . . . . . . . . . . 2755

Wav-BERT: Cooperative Acoustic and Linguistic Representation Learning for Low-Resource Speech Recognition

Guolin Zheng, Yubei Xiao, Ke Gong, Pan Zhou, Xiaodan Liang and Liang Lin

Multilingual AMR Parsing with Noisy Knowledge Distillation

Deng Cai, Xin Li, Jackie Chun-Sing Ho, Lidong Bing and Wai Lam . . . . . . . . . . . . . . . 2778

Open-Domain Contextual Link Prediction and its Complementarity with Entailment Graphs

Mohammad Javad Hosseini, Shay B. Cohen, Mark Johnson and Mark Steedman . . . . . . . . . . 2790

Analysis of Language Change in Collaborative Instruction Following

Anna Effenberger, Rhia Singh, Eva Yan, Alane Suhr and Yoav Artzi . .

Counter-Interference Adapter for Multilingual Machine Translation

Yaoming Zhu, Jiangtao Feng, Chengqi Zhao, Mingxuan Wang and Lei Li . .

2812

Progressive Transformer-Based Generation of Radiology Reports

Farhad Nooralahzadeh, Nicolas Perez Gonzalez, Thomas Frauenfelder, Koji Fujimoto and Michael

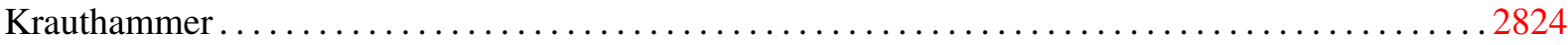

"Be nice to your wife! The restaurants are closed": Can Gender Stereotype Detection Improve Sexism Classification?

Patricia Chiril, Farah Benamara and Véronique Moriceau.

Automatic Discrimination between Inherited and Borrowed Latin Words in Romance Languages

Alina Maria Cristea, Liviu P. Dinu, Simona Georgescu, Mihnea-Lucian Mihai and Ana Sabina Uban 2845

Adapting Language Models for Zero-shot Learning by Meta-tuning on Dataset and Prompt Collections

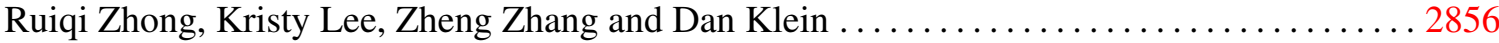

Knowledge-Interactive Network with Sentiment Polarity Intensity-Aware Multi-Task Learning for Emotion Recognition in Conversations

Yunhe Xie, Kailai Yang, Chengjie Sun, Bingquan Liu and Zhenzhou Ji ...

Minimizing Annotation Effort via Max-Volume Spectral Sampling

Ariadna Quattoni and Xavier Carreras 2890

On the Complementarity between Pre-Training and Back-Translation for Neural Machine Translation

Xuebo Liu, Longyue Wang, Derek F. Wong, Liang Ding, Lidia S. Chao, Shuming Shi and Zhaopeng

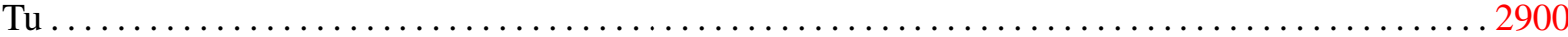

Lexicon-Based Graph Convolutional Network for Chinese Word Segmentation

Kaiyu Huang, Hao Yu, Junpeng Liu, Wei Liu, Jingxiang Cao and Degen Huang . . . . . . . . . 2908

KFCNet: Knowledge Filtering and Contrastive Learning for Generative Commonsense Reasoning

Haonan Li, Yeyun Gong, Jian Jiao, Ruofei Zhang, Timothy Baldwin and Nan Duan . . . . . . . 2918 
Monolingual and Cross-Lingual Acceptability Judgments with the Italian CoLA corpus

Daniela Trotta, Raffaele Guarasci, Elisa Leonardelli and Sara Tonelli ................. 2929

Hyperbolic Hierarchy-Aware Knowledge Graph Embedding for Link Prediction

Zhe Pan and Peng Wang .

A Discourse-Aware Graph Neural Network for Emotion Recognition in Multi-Party Conversation

Yang Sun, Nan Yu and Guohong Fu ....................................... 2949

MeLT: Message-Level Transformer with Masked Document Representations as Pre-Training for Stance Detection

Matthew Matero, Nikita Soni, Niranjan Balasubramanian and H. Andrew Schwartz ........ 2959

LMSOC: An Approach for Socially Sensitive Pretraining

Vivek Kulkarni, Shubhanshu Mishra and Aria Haghighi ...

Extract, Integrate, Compete: Towards Verification Style Reading Comprehension

Chen Zhang, Yuxuan Lai, Yansong Feng and Dongyan Zhao....................... 2976

Comparing learnability of two dependency schemes: 'semantic' (UD) and 'syntactic' (SUD)

Ryszard Tuora, Adam Przepiórkowski and Aleksander Leczkowski ..................... 2987

Argumentation-Driven Evidence Association in Criminal Cases

Yefei Teng and WenHan Chao

Eliminating Sentiment Bias for Aspect-Level Sentiment Classification with Unsupervised Opinion Extraction

Bo Wang, Tao Shen, Guodong Long, Tianyi Zhou and Yi Chang ................... 3002

Data Efficient Masked Language Modeling for Vision and Language

Yonatan Bitton, Michael Elhadad, Gabriel Stanovsky and Roy Schwartz . . .

Improving Multilingual Neural Machine Translation with Auxiliary Source Languages

Weijia Xu, Yuwei Yin, Shuming Ma, Dongdong Zhang and Haoyang Huang . . . . . . . . . . 3029

How Does Fine-tuning Affect the Geometry of Embedding Space: A Case Study on Isotropy

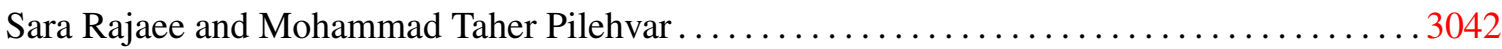

Locality Preserving Sentence Encoding

Changrong Min, Yonghe Chu, Liang Yang, Bo Xu and Hongfei Lin

Knowledge Representation Learning with Contrastive Completion Coding

Bo Ouyang, Wenbing Huang, Runfa Chen, Zhixing Tan, Yang Liu, Maosong Sun and Jihong Zhu 3061

Knowledge-Enhanced Evidence Retrieval for Counterargument Generation

Yohan Jo, Haneul Yoo, JinYeong Bak, Alice Oh, Chris Reed and Eduard Hovy

Investigating Numeracy Learning Ability of a Text-to-Text Transfer Model

Kuntal Kumar Pal and Chitta Baral ...

Modeling Mathematical Notation Semantics in Academic Papers

Hwiyeol Jo, Dongyeop Kang, Andrew Head and Marti A. Hearst . . 
Unpacking the Interdependent Systems of Discrimination: Ableist Bias in NLP Systems through an Intersectional Lens

Saad Hassan, Matt Huenerfauth and Cecilia Ovesdotter Alm ......................... 3116

Constructing Emotional Consensus and Utilizing Unpaired Data for Empathetic Dialogue Generation

Lei Shen, Jinchao Zhang, Jiao Ou, Xiaofang Zhao and Jie Zhou .

Automatic rule generation for time expression normalization

Wentao Ding, Jianhao Chen, Jinmao Li and Yuzhong Qu ...

RW-KD: Sample-wise Loss Terms Re-Weighting for Knowledge Distillation

Peng Lu, Abbas Ghaddar, Ahmad Rashid, Mehdi Rezagholizadeh, Ali Ghodsi and Philippe Langlais 3145

Visual Cues and Error Correction for Translation Robustness

Zhenhao Li, Marek Rei and Lucia Specia

Beyond the Tip of the Iceberg: Assessing Coherence of Text Classifiers

Shane Storks and Joyce Chai ...

Does Pretraining for Summarization Require Knowledge Transfer?

Kundan Krishna, Jeffrey Bigham and Zachary C. Lipton.

Bandits Don't Follow Rules: Balancing Multi-Facet Machine Translation with Multi-Armed Bandits

Julia Kreutzer, David Vilar and Artem Sokolov ............................... 3190

Sometimes We Want Ungrammatical Translations

Prasanna Parthasarathi, Koustuv Sinha, Joelle Pineau and Adina Williams................ 3205

An animated picture says at least a thousand words: Selecting Gif-based Replies in Multimodal Dialog

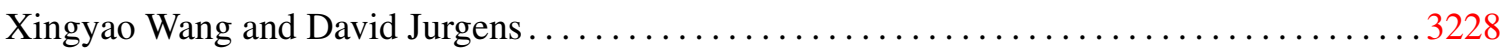

SciCap: Generating Captions for Scientific Figures

Ting-Yao Hsu, C Lee Giles and Ting-Hao Huang ...

SentNoB: A Dataset for Analysing Sentiment on Noisy Bangla Texts

Khondoker Ittehadul Islam, Sudipta Kar, Md Saiful Islam and Mohammad Ruhul Amin ..... 3265

Translate \& Fill: Improving Zero-Shot Multilingual Semantic Parsing with Synthetic Data

Massimo Nicosia, Zhongdi Qu and Yasemin Altun...

NewsBERT: Distilling Pre-trained Language Model for Intelligent News Application

Chuhan Wu, Fangzhao Wu, Yang Yu, Tao Qi, Yongfeng Huang and Qi Liu ...

SD-QA: Spoken Dialectal Question Answering for the Real World

Fahim Faisal, Sharlina Keshava, Md Mahfuz Ibn Alam and Antonios Anastasopoulos ...

The Low-Resource Double Bind: An Empirical Study of Pruning for Low-Resource Machine Translation

Orevaoghene Ahia, Julia Kreutzer and Sara Hooker .......................... 3316

Transformer over Pre-trained Transformer for Neural Text Segmentation with Enhanced Topic Coherence

Kelvin Lo, Yuan Jin, Weicong Tan, Ming Liu, Lan Du and Wray Buntine ............... 3334

Self-Supervised Neural Topic Modeling

Seyed Ali Bahrainian, Martin Jaggi and Carsten Eickhoff. 
Coreference-aware Surprisal Predicts Brain Response

Evan Jaffe, Byung-Doh Oh and William Schuler ...

Distilling the Knowledge of Large-scale Generative Models into Retrieval Models for Efficient Opendomain Conversation

Beomsu Kim, Seokjun Seo, Seungju Han, Enkhbayar Erdenee and Buru Chang ...

Modeling Users and Online Communities for Abuse Detection: A Position on Ethics and Explainability

Pushkar Mishra, Helen Yannakoudakis and Ekaterina Shutova ...................... 3374

Detecting Community Sensitive Norm Violations in Online Conversations

Chan Young Park, Julia Mendelsohn, Karthik Radhakrishnan, Kinjal Jain, Tushar Kanakagiri, David

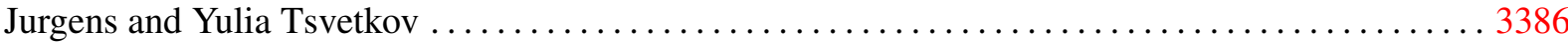

SupCL-Seq: Supervised Contrastive Learning for Downstream Optimized Sequence Representations

Hooman Sedghamiz, Shivam Raval, Enrico Santus, Tuka Alhanai and Mohammad Ghassemi 3398

mDAPT: Multilingual Domain Adaptive Pretraining in a Single Model

Rasmus Kær Jørgensen, Mareike Hartmann, Xiang Dai and Desmond Elliott ... 3404

COSMic: A Coherence-Aware Generation Metric for Image Descriptions

Mert Inan, Piyush Sharma, Baber Khalid, Radu Soricut, Matthew Stone and Malihe Alikhani 3419

Relation-Guided Pre-Training for Open-Domain Question Answering

Ziniu Hu, Yizhou Sun and Kai-Wei Chang ... 3431

MURAL: Multimodal, Multitask Representations Across Languages

Aashi Jain, Mandy Guo, Krishna Srinivasan, Ting Chen, Sneha Kudugunta, Chao Jia, Yinfei Yang

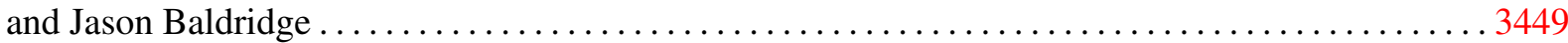

AStitchInLanguageModels: Dataset and Methods for the Exploration of Idiomaticity in Pre-Trained Language Models

Harish Tayyar Madabushi, Edward Gow-Smith, Carolina Scarton and Aline Villavicencio ... 3464

Refine and Imitate: Reducing Repetition and Inconsistency in Persuasion Dialogues via Reinforcement Learning and Human Demonstration

Weiyan Shi, Yu Li, Saurav Sahay and Zhou Yu 3478

A Computational Exploration of Pejorative Language in Social Media

Liviu P. Dinu, Ioan-Bogdan Iordache, Ana Sabina Uban and Marcos Zampieri ...

Evidence-based Fact-Checking of Health-related Claims

Mourad Sarrouti, Asma Ben Abacha, Yassine Mrabet and Dina Demner-Fushman

Learning and Analyzing Generation Order for Undirected Sequence Models

Yichen Jiang and Mohit Bansal ...

Automatic Bilingual Markup Transfer

Thomas Zenkel, Joern Wuebker and John DeNero

Exploring a Unified Sequence-To-Sequence Transformer for Medical Product Safety Monitoring in Social Media

Shivam Raval, Hooman Sedghamiz, Enrico Santus, Tuka Alhanai, Mohammad Ghassemi and Em-

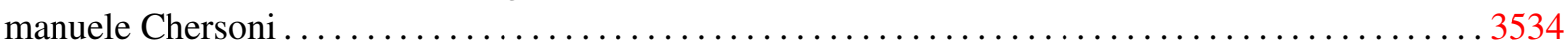


Disentangling Generative Factors in Natural Language with Discrete Variational Autoencoders

Giangiacomo Mercatali and André Freitas . .............................. 3547

MSD: Saliency-aware Knowledge Distillation for Multimodal Understanding

Woojeong Jin, Maziar Sanjabi, Shaoliang Nie, Liang Tan, Xiang Ren and Hamed Firooz . . . 3557

Do UD Trees Match Mention Spans in Coreference Annotations?

Martin Popel, Zdeněk Žabokrtský, Anna Nedoluzhko, Michal Novák and Daniel Zeman . . . . 3570

Beyond Distillation: Task-level Mixture-of-Experts for Efficient Inference

Sneha Kudugunta, Yanping Huang, Ankur Bapna, Maxim Krikun, Dmitry Lepikhin, Minh-Thang

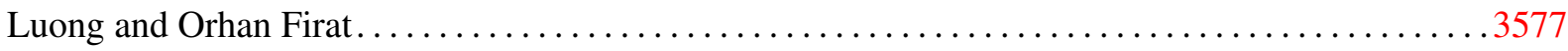

TAG: Gradient Attack on Transformer-based Language Models

Jieren Deng, Yijue Wang, Ji Li, Chenghong Wang, Chao Shang, Hang Liu, Sanguthevar Rajasekaran

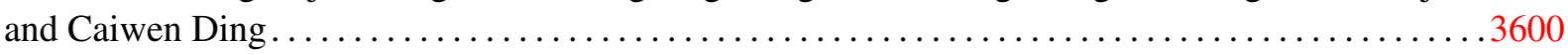

Generating Realistic Natural Language Counterfactuals

Marcel Robeer, Floris Bex and Ad Feelders ................................. 3611

Unsupervised Chunking as Syntactic Structure Induction with a Knowledge-Transfer Approach

Anup Anand Deshmukh, Qianqiu Zhang, Ming Li, Jimmy Lin and Lili Mou..............3626

Model-based analysis of brain activity reveals the hierarchy of language in 305 subjects

Charlotte Caucheteux, Alexandre Gramfort and Jean-Remi King .................... 3635

Gated Transformer for Robust De-noised Sequence-to-Sequence Modelling

Ayan Sengupta, Amit Kumar, Sourabh Kumar Bhattacharjee and Suman Roy . .

Token-wise Curriculum Learning for Neural Machine Translation

Chen Liang, Haoming Jiang, Xiaodong Liu, Pengcheng He, Weizhu Chen, Jianfeng Gao and Tuo

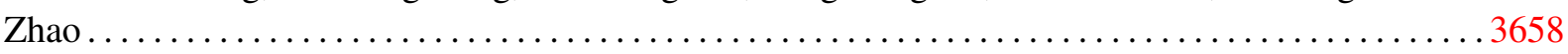

RelDiff: Enriching Knowledge Graph Relation Representations for Sensitivity Classification

Hitarth Narvala, Graham McDonald and Iadh Ounis ............................ 3671

Post-Editing Extractive Summaries by Definiteness Prediction

Jad Kabbara and Jackie Chi Kit Cheung ................................. 3682

Leveraging Pretrained Models for Automatic Summarization of Doctor-Patient Conversations

Longxiang Zhang, Renato Negrinho, Arindam Ghosh, Vasudevan Jagannathan, Hamid Reza Hassanzadeh, Thomas Schaaf and Matthew R. Gormley ................................. 3693

Distilling Knowledge for Empathy Detection

Mahshid Hosseini and Cornelia Caragea ................................... 3713

Adapting Entities across Languages and Cultures

Denis Peskov, Viktor Hangya, Jordan Boyd-Graber and Alexander Fraser ............... 3725

ODIST: Open World Classification via Distributionally Shifted Instances

Lei Shu, Yassine Benajiba, Saab Mansour and Yi Zhang ............................. 3751

LAMAD: A Linguistic Attentional Model for Arabic Text Diacritization

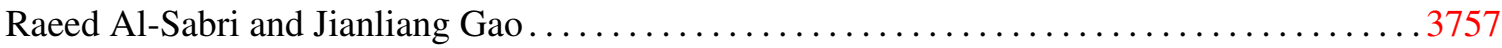


Sequence-to-Lattice Models for Fast Translation

Yuntian Deng and Alexander Rush

Towards Realistic Single-Task Continuous Learning Research for NER

Justin Payan, Yuval Merhav, He Xie, Satyapriya Krishna, Anil Ramakrishna, Mukund Sridhar and

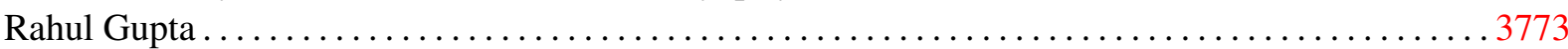

Retrieval Augmentation Reduces Hallucination in Conversation

Kurt Shuster, Spencer Poff, Moya Chen, Douwe Kiela and Jason Weston ...

.3784

Towards Automatic Bias Detection in Knowledge Graphs

Daphna Keidar, Mian Zhong, Ce Zhang, Yash Raj Shrestha and Bibek Paudel ...

Searching for More Efficient Dynamic Programs

Tim Vieira, Ryan Cotterell and Jason Eisner.

Revisiting Robust Neural Machine Translation: A Transformer Case Study

Peyman Passban, Puneeth Saladi and Qun Liu ...

Can NLI Models Verify QA Systems' Predictions?

Jifan Chen, Eunsol Choi and Greg Durrett ..

Parameter-Efficient Domain Knowledge Integration from Multiple Sources for Biomedical Pre-trained Language Models

Qiuhao Lu, Dejing Dou and Thien Huu Nguyen

Uncovering Implicit Gender Bias in Narratives through Commonsense Inference

Tenghao Huang, Faeze Brahman, Vered Shwartz and Snigdha Chaturvedi ............... 3866

Contrastive Document Representation Learning with Graph Attention Networks

Peng Xu, Xinchi Chen, Xiaofei Ma, Zhiheng Huang and Bing Xiang

Convex Aggregation for Opinion Summarization

Hayate Iso, Xiaolan Wang, Yoshihiko Suhara, Stefanos Angelidis and Wang-Chiew Tan ..... 3885

Using Optimal Transport as Alignment Objective for fine-tuning Multilingual Contextualized Embeddings

Sawsan Alqahtani, Garima Lalwani, Yi Zhang, Salvatore Romeo and Saab Mansour ........ 3904

Uncertainty-Aware Machine Translation Evaluation

Taisiya Glushkova, Chrysoula Zerva, Ricardo Rei and André F. T. Martins

Neural Unification for Logic Reasoning over Natural Language

Gabriele Picco, Thanh Lam Hoang, Marco Luca Sbodio and Vanessa Lopez . . .

From None to Severe: Predicting Severity in Movie Scripts

Yigeng Zhang, Mahsa Shafaei, Fabio Gonzalez and Thamar Solorio ...

Benchmarking Meta-embeddings: What Works and What Does Not

Iker García-Ferrero, Rodrigo Agerri and German Rigau ...

A Plug-and-Play Method for Controlled Text Generation

Damian Pascual, Beni Egressy, Clara Meister, Ryan Cotterell and Roger Wattenhofer

A Corpus-based Syntactic Analysis of Two-termed Unlike Coordination

Julie Kallini and Christiane Fellbaum. ... 
Weakly Supervised Contrastive Learning for Chest X-Ray Report Generation

An Yan, Zexue He, Xing Lu, Jiang Du, Eric Chang, Amilcare Gentili, Julian McAuley and Chun-

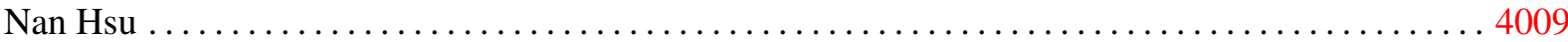

NUANCED: Natural Utterance Annotation for Nuanced Conversation with Estimated Distributions Zhiyu Chen, Honglei Liu, Hu Xu, Seungwhan Moon, Hao Zhou and Bing Liu .............. 4016

Table-based Fact Verification With Salience-aware Learning

Fei Wang, Kexuan Sun, Jay Pujara, Pedro Szekely and Muhao Chen .. 4025

Detecting Frames in News Headlines and Lead Images in U.S. Gun Violence Coverage

Isidora Tourni, Lei Guo, Taufiq Husada Daryanto, Fabian Zhafransyah, Edward Edberg Halim, Mona Jalal, Boqi Chen, Sha Lai, Hengchang Hu, Margrit Betke, Prakash Ishwar and Derry Tanti Wijaya 4037

Multi-task Learning to Enable Location Mention Identification in the Early Hours of a Crisis Event

Sarthak Khanal and Doina Caragea ....................................... 4051

Graph-Based Decoding for Task Oriented Semantic Parsing

Jeremy Cole, Nanjiang Jiang, Panupong Pasupat, Luheng He and Peter Shaw . .

Expected Validation Performance and Estimation of a Random Variable's Maximum

Jesse Dodge, Suchin Gururangan, Dallas Card, Roy Schwartz and Noah A. Smith . .

How May I Help You? Using Neural Text Simplification to Improve Downstream NLP Tasks

Hoang Van, Zheng Tang and Mihai Surdeanu. .

Subformer: Exploring Weight Sharing for Parameter Efficiency in Generative Transformers

Machel Reid, Edison Marrese-Taylor and Yutaka Matsuo ........................... 4081

Leveraging Information Bottleneck for Scientific Document Summarization

Jiaxin Ju, Ming Liu, Huan Yee Koh, Yuan Jin, Lan Du and Shirui Pan . .

Reconsidering the Past: Optimizing Hidden States in Language Models

Davis Yoshida and Kevin Gimpel

Attend, Memorize and Generate: Towards Faithful Table-to-Text Generation in Few Shots

Wenting Zhao, Ye Liu, Yao Wan and Philip Yu ............................ 4106

ARCH: Efficient Adversarial Regularized Training with Caching

Simiao Zuo, Chen Liang, Haoming Jiang, Pengcheng He, Xiaodong Liu, Jianfeng Gao, Weizhu

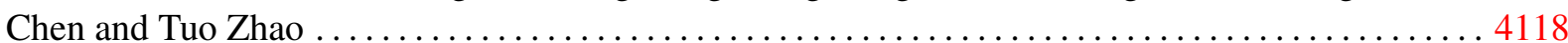

Probing Commonsense Explanation in Dialogue Response Generation

Pei Zhou, Pegah Jandaghi, Hyundong Cho, Bill Yuchen Lin, Jay Pujara and Xiang Ren ...... 4132

NOAHQA: Numerical Reasoning with Interpretable Graph Question Answering Dataset

Qiyuan Zhang, Lei Wang, Sicheng Yu, Shuohang Wang, Yang Wang, Jing Jiang and Ee-Peng Lim 4147

Textual Time Travel: A Temporally Informed Approach to Theory of Mind

Akshatha Arodi and Jackie Chi Kit Cheung...

Detect and Perturb: Neutral Rewriting of Biased and Sensitive Text via Gradient-based Decoding

Zexue He, Bodhisattwa Prasad Majumder and Julian McAuley ...................... 4173 
HyperExpan: Taxonomy Expansion with Hyperbolic Representation Learning

Mingyu Derek Ma, Muhao Chen, Te-Lin Wu and Nanyun Peng ................... 4182

Want To Reduce Labeling Cost? GPT-3 Can Help

Shuohang Wang, Yang Liu, Yichong Xu, Chenguang Zhu and Michael Zeng . .

Written Justifications are Key to Aggregate Crowdsourced Forecasts

Saketh Kotamraju and Eduardo Blanco 4206

Cleaning Dirty Books: Post-OCR Processing for Previously Scanned Texts

Allen Kim, Charuta Pethe, Naoya Inoue and Steve Skiena

Bag of Tricks for Optimizing Transformer Efficiency

Ye Lin, Yanyang Li, Tong Xiao and Jingbo Zhu

Non-Parametric Unsupervised Domain Adaptation for Neural Machine Translation

Xin Zheng, Zhirui Zhang, Shujian Huang, Boxing Chen, Jun Xie, Weihua Luo and Jiajun Chen 4234

The Topic Confusion Task: A Novel Evaluation Scenario for Authorship Attribution

Malik Altakrori, Jackie Chi Kit Cheung and Benjamin C. M. Fung

Micromodels for Efficient, Explainable, and Reusable Systems: A Case Study on Mental Health

Andrew Lee, Jonathan K. Kummerfeld, Larry An and Rada Mihalcea ...

Discovering Explanatory Sentences in Legal Case Decisions Using Pre-trained Language Models

Jaromir Savelka and Kevin Ashley ....................................... 4273

FCM: A Fine-grained Comparison Model for Multi-turn Dialogue Reasoning

Xu Wang, Hainan Zhang, Shuai Zhao, Yanyan Zou, Hongshen Chen, Zhuoye Ding, Bo Cheng and

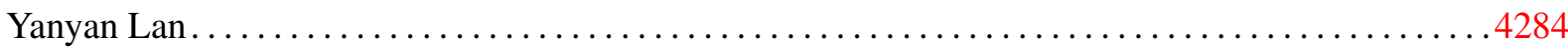

Reference-based Weak Supervision for Answer Sentence Selection using Web Data

Vivek Krishnamurthy, Thuy Vu and Alessandro Moschitti 4294

A Deep Decomposable Model for Disentangling Syntax and Semantics in Sentence Representation

Dingcheng Li, Hongliang Fei, Shaogang Ren and Ping Li ..................... 4300

Improved Word Sense Disambiguation with Enhanced Sense Representations

Yang Song, Xin Cai Ong, Hwee Tou Ng and Qian Lin ...

Rethinking Zero-shot Neural Machine Translation: From a Perspective of Latent Variables

Weizhi Wang, Zhirui Zhang, Yichao Du, Boxing Chen, Jun Xie and Weihua Luo.

FastCorrect 2: Fast Error Correction on Multiple Candidates for Automatic Speech Recognition

Yichong Leng, Xu Tan, Rui Wang, Linchen Zhu, Jin Xu, Wenjie Liu, Linquan Liu, Xiang-Yang Li, Tao Qin, Edward Lin and Tie-Yan Liu ..................................... 4328

Task-Oriented Clustering for Dialogues

Chenxu Lv, Hengtong Lu, Shuyu Lei, Huixing Jiang, Wei Wu, Caixia Yuan and Xiaojie Wang4338

Mitigating Data Poisoning in Text Classification with Differential Privacy

Chang Xu, Jun Wang, Francisco Guzmán, Benjamin Rubinstein and Trevor Cohn .......... 4348

Does Vision-and-Language Pretraining Improve Lexical Grounding?

Tian Yun, Chen Sun and Ellie Pavlick 
Character-based PCFG Induction for Modeling the Syntactic Acquisition of Morphologically Rich Languages

Lifeng Jin, Byung-Doh Oh and William Schuler........................... 4367

Block-wise Word Embedding Compression Revisited: Better Weighting and Structuring

Jong-Ryul Lee, Yong-Ju Lee and Yong-Hyuk Moon. .

Switch Point biased Self-Training: Re-purposing Pretrained Models for Code-Switching

Parul Chopra, Sai Krishna Rallabandi, Alan W Black and Khyathi Raghavi Chandu ...

Influence Tuning: Demoting Spurious Correlations via Instance Attribution and Instance-Driven Updates

Xiaochuang Han and Yulia Tsvetkov

Learning Task Sampling Policy for Multitask Learning

Dhanasekar Sundararaman, Henry Tsai, Kuang-Huei Lee, Iulia Turc and Lawrence Carin . . . 4410

Competing Independent Modules for Knowledge Integration and Optimization

Parsa Bagherzadeh and Sabine Bergler ................................. 4416

An Exploratory Study on Long Dialogue Summarization: What Works and What's Next

Yusen Zhang, Ansong Ni, Tao Yu, Rui Zhang, Chenguang Zhu, Budhaditya Deb, Asli Celikyilmaz, Ahmed Hassan Awadallah and Dragomir Radev ................................... 4426

Improving Text Auto-Completion with Next Phrase Prediction

Dong-Ho Lee, Zhiqiang Hu and Roy Ka-Wei Lee. . .

MOMENTA: A Multimodal Framework for Detecting Harmful Memes and Their Targets

Shraman Pramanick, Shivam Sharma, Dimitar Dimitrov, Md. Shad Akhtar, Preslav Nakov and

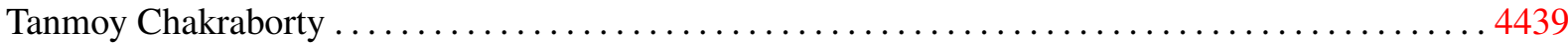

NICE: Neural Image Commenting with Empathy

Kezhen Chen, Qiuyuan Huang, Daniel McDuff, Xiang Gao, Hamid Palangi, Jianfeng Wang, Ken-

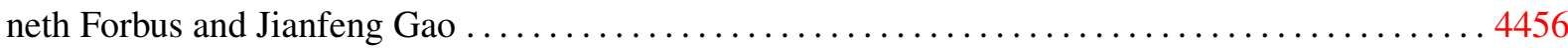

HAConvGNN: Hierarchical Attention Based Convolutional Graph Neural Network for Code Documentation Generation in Jupyter Notebooks

Xuye Liu, Dakuo Wang, April Wang, Yufang Hou and Lingfei Wu

A multilabel approach to morphosyntactic probing

Naomi Shapiro, Amandalynne Paullada and Shane Steinert-Threlkeld ................. 4486

Co-Teaching Student-Model through Submission Results of Shared Task

Kouta Nakayama, Shuhei Kurita, Akio Kobayashi, Yukino Baba and Satoshi Sekine

KLMo: Knowledge Graph Enhanced Pretrained Language Model with Fine-Grained Relationships

Lei He, Suncong Zheng, Tao Yang and Feng Zhang ......................... 4536

Do We Know What We Don't Know? Studying Unanswerable Questions beyond SQuAD 2.0

Elior Sulem, Jamaal Hay and Dan Roth .................................... 4543

Glyph Enhanced Chinese Character Pre-Training for Lexical Sememe Prediction

Boer Lyu, Lu Chen and Kai Yu . . . . . . . . . ............................... 4549

Active Learning for Rumor Identification on Social Media

Parsa Farinneya, Mohammad Mahdi Abdollah Pour, Sardar Hamidian and Mona Diab....... 4556 
Cross-Domain Data Integration for Named Entity Disambiguation in Biomedical Text

Maya Varma, Laurel Orr, Sen Wu, Megan Leszczynski, Xiao Ling and Christopher Ré ..... . 4566

Self-Training using Rules of Grammar for Few-Shot NLU

Joonghyuk Hahn, Hyunjoon Cheon, Kyuyeol Han, Cheongjae Lee, Junseok Kim and Yo-Sub Han 4576

Aspect-based Sentiment Analysis in Question Answering Forums

Wenxuan Zhang, Yang Deng, Xin Li, Lidong Bing and Wai Lam...

ForumSum: A Multi-Speaker Conversation Summarization Dataset

Misha Khalman, Yao Zhao and Mohammad Saleh ........................... 4592

Question Answering over Electronic Devices: A New Benchmark Dataset and a Multi-Task Learning based QA Framework

Abhilash Nandy, Soumya Sharma, Shubham Maddhashiya, Kapil Sachdeva, Pawan Goyal and

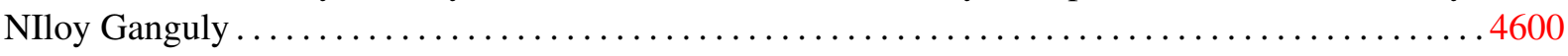

Comprehensive Punctuation Restoration for English and Polish

Michał Pogoda and Tomasz Walkowiak. 4610

Syntactically Diverse Adversarial Network for Knowledge-Grounded Conversation Generation

Fuwei Cui, Hui Di, Hongjie Ren, Kazushige Ouchi, Ze Liu and Jinan Xu .............. 4620

QACE: Asking Questions to Evaluate an Image Caption

Hwanhee Lee, Thomas Scialom, Seunghyun Yoon, Franck Dernoncourt and Kyomin Jung . . 4631

Secoco: Self-Correcting Encoding for Neural Machine Translation

Tao Wang, Chengqi Zhao, Mingxuan Wang, Lei Li, Hang Li and Deyi Xiong............. 4639

Simple or Complex? Complexity-controllable Question Generation with Soft Templates and Deep Mixture of Experts Model

Sheng Bi, Xiya Cheng, Yuan-Fang Li, Lizhen Qu, Shirong Shen, Guilin Qi, Lu Pan and Yinlin

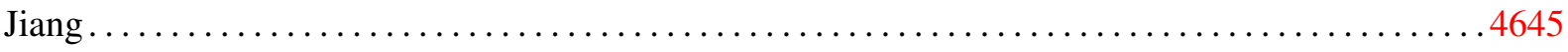

Predicting Anti-Asian Hateful Users on Twitter during COVID-19

Jisun An, Haewoon Kwak, Claire Seungeun Lee, Bogang Jun and Yong-Yeol Ahn . . .

Fine-grained Typing of Emerging Entities in Microblogs

Satoshi Akasaki, Naoki Yoshinaga and Masashi Toyoda ...

Data-Efficient Language Shaped Few-shot Image Classification

Zhenwen Liang and Xiangliang Zhang....

Beyond Glass-Box Features: Uncertainty Quantification Enhanced Quality Estimation for Neural Machine Translation

Ke Wang, Yangbin Shi, Jiayi Wang, Yuqi Zhang, Yu Zhao and Xiaolin Zheng 4687

Fight Fire with Fire: Fine-tuning Hate Detectors using Large Samples of Generated Hate Speech

Tomer Wullach, Amir Adler and Einat Minkov .................................. 4699

AutoEQA: Auto-Encoding Questions for Extractive Question Answering

Stalin Varanasi, Saadullah Amin and Guenter Neumann

A Multi-label Multi-hop Relation Detection Model based on Relation-aware Sequence Generation

Linhai Zhang, Deyu Zhou, Chao Lin and Yulan He .......................... 4713 
Don't Discard All the Biased Instances: Investigating a Core Assumption in Dataset Bias Mitigation Techniques

Hossein Amirkhani and Mohammad Taher Pilehvar. .

Stacked AMR Parsing with Silver Data

Qingrong Xia, Zhenghua Li, Rui Wang and Min Zhang...

Speculative Sampling in Variational Autoencoders for Dialogue Response Generation

Shoetsu Sato, Naoki Yoshinaga, Masashi Toyoda and Masaru Kitsuregawa ...

Perceived and Intended Sarcasm Detection with Graph Attention Networks

Joan Plepi and Lucie Flek

Contrastive Representation Learning for Exemplar-Guided Paraphrase Generation

Haoran Yang, Wai Lam and Piji Li ...

MAD-G: Multilingual Adapter Generation for Efficient Cross-Lingual Transfer

Alan Ansell, Edoardo Maria Ponti, Jonas Pfeiffer, Sebastian Ruder, Goran Glavaš, Ivan Vulić and

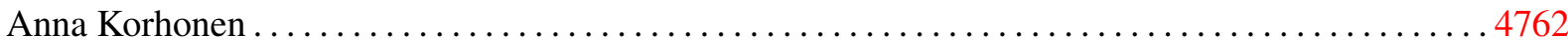

Sustainable Modular Debiasing of Language Models

Anne Lauscher, Tobias Lueken and Goran Glavaš . . .

A Divide-And-Conquer Approach for Multi-label Multi-hop Relation Detection in Knowledge Base Question Answering

Deyu Zhou, Yanzheng Xiang, Linhai Zhang, Chenchen Ye, Qian-Wen Zhang and Yunbo Cao 4798

Counterfactual Adversarial Learning with Representation Interpolation

Wei Wang, Boxin Wang, Ning Shi, Jinfeng Li, Bingyu Zhu, Xiangyu Liu and Rong Zhang... 4809

'Just What do You Think You're Doing, Dave?' A Checklist for Responsible Data Use in NLP

Anna Rogers, Timothy Baldwin and kobi leins ...

Counter-Contrastive Learning for Language GANs

Yekun Chai, Haidong Zhang, Qiyue Yin and Junge Zhang ...

Incorporating Circumstances into Narrative Event Prediction

Shichao Wang, Xiangrui Cai, HongBin Wang and Xiaojie Yuan

MultiFix: Learning to Repair Multiple Errors by Optimal Alignment Learning

HyeonTae Seo, Yo-Sub Han and Sang-Ki Ko . .

HOTTER: Hierarchical Optimal Topic Transport with Explanatory Context Representations

Sabine Wehnert, Christian Scheel, Simona Szakács-Behling, Maret Nieländer, Patrick Mielke and Ernesto William De Luca ........................................... 4856

Grammatical Error Correction with Contrastive Learning in Low Error Density Domains

Hannan Cao, Wenmian Yang and Hwee Tou Ng .

Improving Unsupervised Commonsense Reasoning Using Knowledge-Enabled Natural Language Inference

Canming Huang, Weinan He and Yongmei Liu ...

Does Putting a Linguist in the Loop Improve NLU Data Collection?

Alicia Parrish, William Huang, Omar Agha, Soo-Hwan Lee, Nikita Nangia, Alexia Warstadt, Karmanya Aggarwal, Emily Allaway, Tal Linzen and Samuel R. Bowman. 4886 
Tiered Reasoning for Intuitive Physics: Toward Verifiable Commonsense Language Understanding

Shane Storks, Qiaozi Gao, Yichi Zhang and Joyce Chai ........................ 4902

Making Heads and Tails of Models with Marginal Calibration for Sparse Tagsets

Michael Kranzlein, Nelson F. Liu and Nathan Schneider. .

GeDi: Generative Discriminator Guided Sequence Generation

Ben Krause, Akhilesh Deepak Gotmare, Bryan McCann, Nitish Shirish Keskar, Shafiq Joty, Richard Socher and Nazneen Fatema Rajani . . . . . . . . . . . . . . . . . . . . . . . . . . . . . . . . . . . . 4929 